\title{
Phenotypic factors influencing the variation in response of circulating cholesterol level to personalised dietary advice in the Food4Me study
}

\author{
Laura Kirwan ${ }^{1}$, Marianne C. Walsh ${ }^{1}$, Carlos Celis-Morales ${ }^{2}$, Cyril F. M. Marsaux ${ }^{3}$, \\ Katherine M. Livingstone ${ }^{2}$, Santiago Navas-Carretero ${ }^{4,5}$, Rosalind Fallaize ${ }^{6}$, Clare B. O’Donovan ${ }^{1}$,
} Clara Woolhead $^{1}$, Hannah Forster ${ }^{1}$, Silvia Kolossa ${ }^{7}$, Hannelore Daniel ${ }^{7}$, George Moschonis ${ }^{8}$, Yannis Manios ${ }^{8}$, Agnieszka Surwillo9, Magdalena Godlewska', Iwona Traczyk ${ }^{9}$, Christian A. Drevon ${ }^{10}$, Mike J. Gibney ${ }^{1}$, Julie A. Lovegrove ${ }^{6}$, J. Alfredo Martinez ${ }^{4,5}$, Wim H. M. Saris ${ }^{3}$, John C. Mathers ${ }^{2}$, Eileen R. Gibney ${ }^{1}$ and Lorraine Brennan ${ }^{1 *}$ on behalf of the Food4me study

${ }^{1}$ UCD School of Agriculture and Food Science, UCD Institute of Food and Health, University College Dublin, Belfield, Dublin 4, Republic of Ireland

${ }^{2}$ Human Nutrition Research Centre, Institute of Cellular Medicine, Newcastle University, Newcastle Upon Tyne NE4 5PL, UK

${ }^{3}$ Department of Human Biology, NUTRIM School of Nutrition and Translational Research in Metabolism, Maastricht University Medical Centre + (MUMC+), Maastricht, The Netherlands

${ }^{4}$ Department of Nutrition, Food Science and Physiology, Centre for Nutrition Research, University of Navarra, Pamplona, Spain ${ }^{5}$ CIBER Fisiopatogía de la Obesidad y Nutrición (CIBERobn), Instituto de Salud Carlos III, Madrid, Spain

${ }^{6}$ Hugh Sinclair Unit of Human Nutrition and Institute for Cardiovascular and Metabolic Research, University of Reading, Reading, $U K$

${ }^{7}$ ZIEL Research Center of Nutrition and Food Sciences, Biochemistry Unit, Technische Universität München, München, Germany

${ }^{8}$ Department of Nutrition and Dietetics, Harokopio University, Athens, Greece

${ }^{9}$ National Food E Nutrition Institute (IZZ), Warsaw, Poland

${ }^{10}$ Department of Nutrition, Institute of Basic Medical Sciences, Faculty of Medicine, University of Oslo, Oslo, Norway

(Submitted 15 April 2016 - Final revision received 21 October 2016 - Accepted 10 November 2016 - First published online 9 January 2017)

\section{Abstract}

Individual response to dietary interventions can be highly variable. The phenotypic characteristics of those who will respond positively to personalised dietary advice are largely unknown. The objective of this study was to compare the phenotypic profiles of differential responders to personalised dietary intervention, with a focus on total circulating cholesterol. Subjects from the Food4Me multi-centre study were classified as responders or non-responders to dietary advice on the basis of the change in cholesterol level from baseline to month 6 , with lower and upper quartiles defined as responder and non-responder groups, respectively. There were no significant differences between demographic and anthropometric profiles of the groups. Furthermore, with the exception of alcohol, there was no significant difference in reported dietary intake, at baseline. However, there were marked differences in baseline fatty acid profiles. The responder group had significantly higher levels of stearic acid (18:0, $P=0.034)$ and lower levels of palmitic acid $(16: 0, P=0.009)$. Total MUFA $(P=0.016)$ and total PUFA $(P=0.008)$ also differed between the groups. In a step-wise logistic regression model, age, baseline total cholesterol, glucose, five fatty acids and alcohol intakes were selected as factors that successfully discriminated responders from non-responders, with sensitivity of $82 \%$ and specificity of $83 \%$. The successful delivery of personalised dietary advice may depend on our ability to identify phenotypes that are responsive. The results demonstrate the potential use of metabolic profiles in identifying response to an intervention and could play an important role in the development of precision nutrition.

Key words: Personalised nutrition: Cholesterol: Responders: Phenotypes: Fatty acid profiles

At a population level, generic dietary advice is provided using a 'one-size-fits-all' approach on the basis of requirements for population groups ${ }^{(1)}$, which ignores inter-individual differences, and therefore nutrient requirements. In addition, individuals' responses to dietary interventions can be highly variable ${ }^{(2-4)}$. Demographic characteristics such as sex and age, and factors

Abbreviation: FA, fatty acid.

* Corresponding author: L. Brennan, email lorraine.brennan@ucd.ie 
such as adiposity, physical activity, metabolic profile and genetic factors contribute to this variation ${ }^{(5)}$. This phenomenon is well recognised in the medical field with a current emphasis on precision medicine ${ }^{(6)}$. Considering the reported variation in response to dietary interventions, there is now an emerging recognition that this should be considered in the development of personalised or precision nutrition ${ }^{(7,8)}$. Personalised nutrition or dietary advice that has been tailored for an individual offers the possibility of improving health and reducing risk of diet-related diseases ${ }^{(9)}$. Many studies suggest that tailored dietary advice is more effective than generic advice, promoting greater improvements in dietary behaviours and related health outcomes such as body weight $^{(9,10)}$. A recent meta-analysis reported that personalised interventions were more effective than non-personalised advice, with participants receiving the personalised intervention reducing body weight by $1.8 \mathrm{~kg}$ more on average than those receiving the non-personalised advice ${ }^{(9)}$. However, these studies have not taken individual variability into account, and in the long term the effectiveness of the personalised dietary advice will depend on the ability to tailor advice taking into account knowledge about an individual's potential response to the intervention ${ }^{(11)}$.

The concept of using metabolic profiles to identify responders to dietary interventions is relatively new ${ }^{(7)}$. However, a number of examples exist in the literature demonstrating the potential of such an approach. O'Sullivan et al. ${ }^{(12)}$ used k-means cluster analysis to identify responders and non-responders to a vitamin D intervention. van Bochove et al. ${ }^{(13)}$ applied k-means clustering to lipoprotein profiles and identified three clusters, two of which responded positively to fenofibrate, whereas Elnenaei et al. ${ }^{(14)}$ identified responders and non-responders to vitamin $\mathrm{D}$ and $\mathrm{Ca}$ supplementation, on the basis of a baseline metabolomic profile. Metabolomic and transcriptomic profiles have also been used to discriminate between responders and non-responders to an $n-3$ PUFA supplementation ${ }^{(15)}$. The objective of this study was to investigate differences in the phenotype and in particular blood lipids of responders and non-responders to personalised nutrition, with a specific focus on changes in circulating cholesterol levels. Using data from the Food4Me personalised dietary intervention study, individuals with borderline high baseline total cholesterol $(>5 \mathrm{mmol} / \mathrm{l})$ were examined for factors that predict their response to the intervention.

\section{Methods}

Subjects were participants of the Food4Me study - a 6-month, web-based randomised controlled trial conducted in seven European countries. The aim of the present study was to determine whether providing personalised dietary advice leads to improvements in dietary intakes and health outcomes relative to population-based public health messages. The 1607 adult subjects were randomly assigned to one of four intervention treatment groups - level 0 (standard non-personalised dietary and physical activity guidelines), level 1 (personalised advice based on current diet and physical activity), level 2 (personalised advice based on current diet, physical activity and phenotype) and level 3 (personalised advice based on current diet, physical activity, phenotype and genotype $)^{(16)}$. The control group received conventional, non-personalised advice, and therefore were not considered for this analysis. The study protocol is detailed in the study by Celis-Morales et al. ${ }^{(16)}$.

All data were collected remotely following standardised operating procedures. At baseline, participants received study kits by post containing all necessary materials to perform measurements at home. Printed instructions were included, and demonstration videos were available on the Food4Me website (http://www.food4me.org). Following measurements at baseline and 3 months, participants received a personalised report. The personalised feedback provided was based on a predefined set of algorithms, including anthropometric, physical activity (levels 1-3), phenotypic (levels 2 and 3) and genotypic (level 3 only) data ${ }^{(16)}$.

\section{Demographic characteristics}

The measurement of characteristics including age, country and sex and have been described elsewhere ${ }^{(16)}$. Having excluded the control group and those with normal total cholesterol levels at baseline (total cholesterol $<5 \mathrm{mmol} / \mathrm{l}$ ), there were 151 males and 162 females, with a mean age of 46.8 years from seven European countries - Germany ( $n$ 67), Greece ( $n$ 48), Ireland ( $n$ 39), the Netherlands ( $n$ 54), Poland ( $n$ 30), Spain ( $n$ 43) and the UK ( $n$ 32). Subjects were classified as responders and nonresponders on the basis of the change in blood cholesterol from baseline to month 6 . To achieve this, the subjects were first stratified into quartiles on the basis of cholesterol response; two of the groups, the lower and upper quartiles, were defined as responders and non-responders, respectively. This resulted in seventy-eight responders and seventy-nine non-responders.

\section{Anthropometric measurements}

Body weight, height and waist circumference were selfmeasured and self-reported by participants via the Internet, as described previously ${ }^{(16)}$. They were provided with clear instructions in text and video format to facilitate accurate measurements, and a validation study demonstrated the reliability of these Internet-based, self-reported anthropometric data $^{(17)}$. Waist circumference was measured at the mid-point between the lower rib and the iliac crest using the same tape measure. Physical activity was self-reported using the Baecke questionnaire online ${ }^{(18,19)}$ on the basis of physical activity during the last month. Physical activity level scores were calculated at baseline and month 6 , according to the questionnaire protocol.

\section{Dietary intake measurements}

Habitual dietary intake was quantified using an online FFQ including food items frequently consumed in each of the seven recruitment sites. The Food 4 me FFQ has been compared with a paper-based $\mathrm{FFQ}^{(20)}$ and 4-d weighed food record ${ }^{(21)}$ for both food group and nutrient intakes. The Bland-Altman analysis showed good agreement between the online and the paper-based FFQ for both nutrient and food groups level. Cross-classification into exact plus adjacent quartiles ranged 
from 77 to $97 \%$ at the nutrient level and 77 to $99 \%$ at the food group level. For comparison with the weighed food record, the mean cross-classification into exact agreement plus adjacent was 80 and $78 \%$ for nutrient and food groups, respectively. Importantly, the energy intake estimated by the FFQ was in agreement with the weighed food record, indicating that overall the online FFQ was a suitable tool for assessing dietary intake.

\section{Fatty acid and carotenoid profiles}

Finger-prick blood samples were collected by participants using a test kit provided by Vitas Ltd, as described previously ${ }^{(22)}$. Each participant filled two Dry Blood Spot cards (equivalent to five drops of blood or $150 \mu \mathrm{l}$ of blood per card) at each collection time point. The samples were sent to Vitas (Vitas Ltd) for measurements of total cholesterol, carotenoids and thirty-two fatty acids (FA). The $n-3$ FA index was calculated as the sum of EPA $(20: 5 n-3)$ and DHA $(22: 6 n-3)$. The $\Delta 5$ desaturase index (D5D) and $\Delta 6$ desaturase index (D6D) were calculated on the basis of key enzymes in the metabolism of PUFA. The D5D was calculated as the ratio of arachidonic acid (20:4n-6):dihomo- $\gamma$ linoleic acid (20:3n-6), and the D6D was calculated as the ratio of dihomo- $\gamma$-linoleic acid $(20: 3 n$-6):linoleic acid $(18: 2 n-6)$.

\section{Ethics}

This study was conducted according to the guidelines laid down in the Declaration of Helsinki. The Research Ethical Committees at each participating centre granted ethics approval for the study ${ }^{(16)}$.

\section{Statistical analysis}

The baseline demographic and phenotypic characteristics of responders and non-responders were compared using generalised linear models (GLM). Models were fitted using the GLM (for continuous variables) and GENMOD (a procedure for fitting GLM; for categorical variables) procedures in SAS 9.3 (SAS Institute). To account for multiple comparisons, false discovery rate-adjusted $P$ values are presented for FA profile data.

To assess whether baseline demographic or phenotypic characteristics can discriminate between responders and non-responders, a step-wise logistic regression procedure was applied in four stages. First, only anthropometric characteristics were included (model 1). Next, baseline cholesterol value was added to the model (model 2). Third, dietary intake data were added to the analysis (model 3), and, finally, all demographic, anthropometric, dietary intake and biochemical characteristics were included (model 4). At each stage, the step-wise procedure selected the characteristics that best discriminated between the two groups. Variables were tested using a bootstrapping approach to correct for over-optimism in model fitting. The ability of the models to classify responders and non-responders was assessed using area under the ROC curves. ROC comparisons were performed using a contrast matrix to calculate differences of the areas under the empirical ROC curves.

\section{Results}

\section{Characteristics of responders and non-responders}

Demographic characteristics did not differ significantly between responder and non-responder groups by country $\left(\chi_{6}^{2}=5 \cdot 0\right.$, $P=0.544$, Table 1$)$, sex $\left(\chi_{1}^{2}=0.16, P=0.693\right.$, Table 1$)$ or age $(P=0.082$, Table 1$)$. There was also little difference between responder and non-responder groups with respect to anthropometric characteristics measured at baseline (Table 1).

During the intervention period, both groups significantly reduced BMI, weight and waist circumference, with both groups exhibiting similar effect sizes (Table 1). The responders significantly increased their blood omega- 3 index while the nonresponders did not (mean change $\Delta=0.31 v \cdot 0 \cdot 14, P<0 \cdot 001$ ).

At baseline, the responders and non-responders had similar dietary intakes of most food groups, with the exception of alcohol (Table 2), for which the responders had lower intakes (170 v. $258 \mathrm{~g} / \mathrm{d}, P=0.035)$. After intervention, the responders reported reduced intake of dairy products $(\Delta=-59 \mathrm{~g} / \mathrm{d}$, Table 2$)$, and both responders and non-responders reported significantly reduced red meat intake $(\Delta=-31$ and $-28 \mathrm{~g} / \mathrm{d}$, respectively).

The percentage of participants receiving dietary advice for specific target nutrients was broadly similar (online Supplementary Table S1). The most common nutrient targeted at baseline was salt ( $73 \%$ of responders and $59 \%$ of non-responders). There was no difference in the percentage of responders and nonresponders receiving a dietary message specifically targeted at cholesterol ( $24 v .23 \%, P=0 \cdot 816$ ), although a greater number of non-responders received a message to increase physical activity (56\% of responders $v .73 \%$ of non-responders, $P=0.027$ ). Although the responders had a significant reduction in cholesterol, there was no significant change in physical activity during the intervention period for either group.

At baseline, the responders had higher total cholesterol levels than the non-responders (6.09 v. $5.54 \mathrm{mmol} / 1, P<0.001$, Table 1). The FA profiles differed between the responders and the non-responders at baseline (Table 3). There was no difference between the groups for total SFA $(P=0.203)$, but the responders had lower palmitic acid (16:0, $P=0 \cdot 009)$. At baseline, the responders had significantly lower total MUFA $(P=0.016)$, particularly lower palmitoleic acid (16:1n-7, $P=0.012)$ and $c i s$-vaccenic acid $(18: 1 n-7, P=0 \cdot 001)$. At baseline, the responders had higher total PUFA $(P=0.008)$, particularly linoleic acid $(18: 2 n-6, P=0 \cdot 011)$, eicosadienoic acid (20:2n-6, $P=0.006)$ and DPA (22:5n-3, $P=0 \cdot 014)$. At baseline, both groups had similar carotenoids profiles (Table 4 ).

\section{Discriminating between responders and non-responders}

When the step-wise logistic regression model was applied using demographic and anthropometric data, age and weight were selected as being important factors in discriminating responders from non-responders (model 1, Table 5). The classification accuracy (as measured by the area under the ROC curve, Fig. 1) was 0.61 , indicating that the demographic and anthropometric data do not provide sufficient discriminatory power. As expected, classification accuracy improved when the model was adjusted for baseline cholesterol (model 2, AUC $=0.76$, 
Table 1. Demographic and phenotypic profiles of responders and non-responders $\dagger$

(Numbers and percentages; measurements at baseline and mean change $(\Delta)$ between baseline and month 6 are presented as means with their standard errors)

\begin{tabular}{|c|c|c|c|c|c|c|c|c|c|c|}
\hline & \multicolumn{4}{|c|}{ Responder } & \multicolumn{4}{|c|}{ Non-responder } & \multicolumn{2}{|c|}{ Responder $v$. non-responder } \\
\hline & \multicolumn{2}{|c|}{$n$} & \multicolumn{2}{|c|}{$\%$} & \multicolumn{2}{|c|}{$n$} & \multicolumn{2}{|c|}{$\%$} & $\chi^{2}$ & $P$ \\
\hline Total & \multicolumn{2}{|c|}{78} & & & \multicolumn{2}{|c|}{79} & & & & \\
\hline \multicolumn{11}{|l|}{ Sex } \\
\hline Male & \multicolumn{2}{|c|}{40} & \multicolumn{2}{|c|}{$51 \cdot 20$} & \multicolumn{2}{|c|}{43} & \multicolumn{2}{|c|}{$55 \cdot 13$} & & \\
\hline Female & \multicolumn{2}{|c|}{38} & \multicolumn{2}{|c|}{$48 \cdot 70$} & \multicolumn{2}{|c|}{36} & \multicolumn{2}{|c|}{$46 \cdot 15$} & $0 \cdot 16$ & 0.693 \\
\hline \multicolumn{11}{|l|}{ Country } \\
\hline Germany & \multicolumn{2}{|c|}{19} & \multicolumn{2}{|c|}{24.40} & \multicolumn{2}{|c|}{23} & \multicolumn{2}{|c|}{$29 \cdot 11$} & & \\
\hline Greece & \multirow{2}{*}{\multicolumn{2}{|c|}{10}} & \multicolumn{2}{|c|}{$12 \cdot 80$} & \multicolumn{2}{|c|}{11} & \multicolumn{2}{|c|}{13.92} & & \\
\hline Ireland & & & \multicolumn{2}{|c|}{$14 \cdot 10$} & & & \multicolumn{2}{|c|}{8.86} & & \\
\hline The Netherlands & \multicolumn{2}{|c|}{16} & \multicolumn{2}{|c|}{$20 \cdot 50$} & & & & & & \\
\hline Poland & & & & & & & & & & \\
\hline Spain & & & & & & & & & & \\
\hline UK & & & & & & & & & $5 \cdot 0$ & 0.544 \\
\hline & Bas & line & & & & & & & $P$ fo & \\
\hline & Mean & SEM & Mean & SEM & Mean & SEM & Mean & SEM & Baseline & $\Delta$ \\
\hline Age (years) & $45 \cdot 1$ & 1.35 & & & $48 \cdot 2$ & $1 \cdot 15$ & & & 0.082 & \\
\hline Height $(m)$ & 1.72 & 0.01 & & & 1.74 & 0.01 & & & 0.262 & \\
\hline Weight (kg) & $78 \cdot 1$ & 1.66 & $-1 \cdot 7^{\star}$ & 0.39 & 82.6 & 1.79 & $-1 \cdot 3^{*}$ & 0.37 & 0.065 & 0.429 \\
\hline $\mathrm{BMI}\left(\mathrm{kg} / \mathrm{m}^{2}\right)$ & $26 \cdot 4$ & 0.52 & $-0.6^{*}$ & 0.14 & $27 \cdot 5$ & 0.56 & $-0.4^{*}$ & 0.13 & 0.17 & 0.495 \\
\hline Waist circumference (m) & 0.9 & 0.015 & $-0.02^{*}$ & 0.005 & 0.93 & 0.015 & $-0.02^{*}$ & 0.005 & 0.091 & 0.764 \\
\hline Physical activity level & 1.54 & 0.012 & 0.03 & 0.01 & 1.53 & 0.012 & 0.027 & 0.01 & 0.687 & 0.908 \\
\hline Total cholesterol $(\mathrm{mmol} / \mathrm{l})$ & 6.09 & 0.091 & $-2 \cdot 01^{*}$ & 0.072 & 5.54 & 0.063 & 0.47 & 0.06 & $<0.001^{*}$ & $<0.001^{*}$ \\
\hline Glucose $(\mathrm{mmol} / \mathrm{l})$ & $4 \cdot 13$ & 0.08 & $-0.82^{*}$ & 0.113 & 3.88 & 0.111 & $-0.23^{*}$ & 0.111 & 0.934 & 0.259 \\
\hline Omega-3 index & 5.68 & 0.127 & $0.31^{*}$ & 0.096 & 5.69 & 0.13 & 0.14 & 0.109 & 0.068 & $<0.001^{*}$ \\
\hline
\end{tabular}

* $P$ values are significant at the $5 \%$ level.

$\dagger P$ values were obtained from generalised linear models including the responder group as a factor.

Table 2. Baseline dietary intake $(\mathrm{g} / \mathrm{d})$ and change from baseline to month 6 for responders and non-responders $\dagger$

(Dietary intake at baseline and mean change $(\Delta)$ between baseline and month 6 are presented as means with their standard errors)

\begin{tabular}{|c|c|c|c|c|c|c|c|c|c|c|}
\hline & \multicolumn{4}{|c|}{ Responders } & \multicolumn{4}{|c|}{ Non-responders } & & \\
\hline & \multicolumn{2}{|c|}{ Baseline } & \multicolumn{2}{|c|}{$\Delta$} & \multicolumn{2}{|c|}{ Baseline } & \multicolumn{2}{|c|}{$\Delta$} & \multicolumn{2}{|c|}{$P$ value for difference } \\
\hline & Mean & SEM & Mean & SEM & Mean & SEM & Mean & SEM & Baseline & $\Delta$ \\
\hline Fruit & 445 & 45 & 6 & 33 & 387 & 32 & 37 & 31 & 0.289 & 0.487 \\
\hline Vegetables & 234 & 22 & -6 & 22 & 229 & 14 & 4 & 15 & 0.851 & 0.71 \\
\hline Whole grains & 169 & 18 & 6 & 15 & 127 & 14 & 22 & 12 & 0.064 & 0.413 \\
\hline Oily fish & 23 & 3 & 9 & 6 & 21 & 3 & 4 & 3 & 0.691 & 0.42 \\
\hline Red meat & 95 & 11 & $-31^{*}$ & 10 & 85 & 7 & $-28^{*}$ & 5 & 0.424 & 0.763 \\
\hline Dairy products & 337 & 30 & $-59^{*}$ & 27 & 286 & 28 & -16 & 24 & 0.214 & 0.247 \\
\hline Nuts & 7 & 2 & 0 & 1 & 6 & 1 & 1 & 1 & 0.694 & 0.638 \\
\hline Alcohol & 170 & 23 & -22 & 25 & 258 & 34 & -16 & 35 & $0.035^{\star}$ & 0.892 \\
\hline
\end{tabular}

* Mean changes are significant at the $5 \%$ level.

$\dagger P$ values were obtained from generalised linear models including responder group as a factor.

Table 5, Fig. 1). Including dietary intake data (model 3) did not improve the discriminatory power, with none of the food groups being selected when tested in the step-wise model. When the additional biochemical data were added to the model (model 4), the key variables selected were baseline levels of cholesterol, glucose, stearic acid, DPA and eicosenoic acid, each with significant positive coefficients, and EPA and transFA, each with significant negative coefficients. Alcohol intake also had a significant negative coefficient in this model that included the biochemical variables. The coefficients of the final logistic regression discriminant model are detailed in Table 6. Increases in the variables with positive or negative coefficients were associated with increased or decreased probability of being a responder, respectively. The additional biochemical data significantly improved the classification accuracy (model 4 AUC $=0.90$, Table 5, Fig. 1), with increases in the true-positive rate (sensitivity) resulting in only a small trade-off with the false-positive rate $(1-$ sensitivity). For example, to 
Table 3. Mean percentage of blood total fatty acid at baseline for responders and non-responders and mean change from baseline to month $6 \dagger$

(Fatty acid percentage at baseline and mean change $(\Delta)$ between baseline and month 6 are presented as mean values with their standard errors)

\begin{tabular}{|c|c|c|c|c|c|c|c|c|c|c|c|c|c|}
\hline & \multirow[b]{3}{*}{ Trans-fatty acids } & \multicolumn{4}{|c|}{ Responders } & \multicolumn{4}{|c|}{ Non-responders } & \multicolumn{4}{|c|}{$P$ value for difference } \\
\hline & & \multicolumn{2}{|c|}{ Baseline } & \multicolumn{2}{|c|}{$\Delta$} & \multicolumn{2}{|c|}{ Baseline } & \multicolumn{2}{|c|}{$\Delta$} & \multicolumn{2}{|c|}{ Baseline } & \multicolumn{2}{|c|}{$\Delta$} \\
\hline & & Mean & SEM & Mean & SEM & Mean & SEM & Mean & SEM & $P$ & FDR & $P$ & FDR \\
\hline & & 0.79 & 0.027 & 0.04 & 0.035 & 0.9 & 0.028 & -0.06 & 0.039 & $0.007^{\star}$ & $0.040^{*}$ & 0.059 & 0.295 \\
\hline SFA & Myristic (14:0) & 0.78 & 0.042 & -0.07 & 0.046 & 0.85 & 0.048 & -0.02 & 0.044 & 0.281 & 0.351 & 0.398 & 0.807 \\
\hline SFA & Pentadecyclic (15:0) & 0.21 & 0.006 & 0 & 0.005 & 0.2 & 0.006 & $0.01^{*}$ & 0.006 & 0.166 & 0.239 & 0.046 & 0.295 \\
\hline SFA & Palmitic $(16: 0)$ & 22.89 & 0.157 & -0.04 & 0.188 & 23.63 & 0.229 & -0.46 & 0.36 & $0.009^{*}$ & $0.040^{*}$ & 0.308 & 0.807 \\
\hline SFA & Margaric $(17: 0)$ & 0.32 & 0.005 & $-0.01^{*}$ & 0.006 & 0.31 & 0.008 & -0.01 & 0.009 & 0.799 & 0.799 & 0.617 & 0.807 \\
\hline SFA & Stearic $(18: 0)$ & $12 \cdot 81$ & 0.118 & $0.67^{\star}$ & 0.156 & $12 \cdot 44$ & 0.129 & $0.68^{*}$ & 0.275 & 0.034 & 0.076 & 0.978 & 0.978 \\
\hline SFA & Arachidic $(20: 0)$ & 0.2 & 0.007 & $0.15^{\star}$ & 0.032 & 0.19 & 0.007 & $0.17^{\star}$ & 0.027 & 0.639 & 0.710 & 0.621 & 0.807 \\
\hline MUFA & Palmitoleic $(16: 1 n-7)$ & 1.26 & 0.056 & -0.08 & 0.041 & 1.49 & 0.072 & -0.02 & 0.059 & $0.012^{*}$ & $0.040^{*}$ & 0.436 & 0.807 \\
\hline MUFA & Oleic $n-9(18: 1 n-9)$ & $19 \cdot 21$ & 0.278 & -0.34 & 0.245 & $19 \cdot 9$ & 0.241 & $-0.84^{\star}$ & 0.328 & 0.063 & $0 \cdot 126$ & 0.225 & 0.807 \\
\hline MUFA & cis-Vaccenic $(18: 1 n-7)$ & 1.34 & 0.021 & $0.22^{*}$ & 0.046 & 1.48 & 0.039 & 0.03 & 0.056 & $0.001^{*}$ & $0.020^{*}$ & 0.01 & 0.200 \\
\hline MUFA & Eicosenoic $(20: 1)$ & 0.26 & 0.006 & $-0.01^{*}$ & 0.006 & 0.25 & 0.007 & -0.01 & 0.008 & 0.167 & 0.239 & 0.686 & 0.807 \\
\hline$n-3$ PUFA & $a$-Linolenic $(18: 3 n-3)$ & 0.33 & 0.013 & 0 & 0.017 & 0.34 & 0.018 & 0 & 0.018 & 0.528 & 0.621 & 0.943 & 0.978 \\
\hline$n-3$ PUFA & EPA $(20: 5 n-3)$ & 0.73 & 0.045 & 0.04 & 0.038 & 0.82 & 0.061 & 0.07 & 0.056 & 0.208 & 0.277 & 0.661 & 0.807 \\
\hline$n-3$ PUFA & DPA $(22: 5 n-3)$ & 1.41 & 0.039 & 0.03 & 0.027 & 1.28 & 0.035 & 0.01 & 0.036 & $0.014^{\star}$ & $0.040^{*}$ & 0.67 & 0.807 \\
\hline$n-3$ PUFA & DHA $(22: 6 n-3)$ & 2.96 & 0.1 & $0.31^{*}$ & 0.069 & 3.01 & 0.095 & 0.09 & 0.076 & 0.696 & 0.733 & 0.041 & 0.295 \\
\hline$n-6$ PUFA & Linoleic $(18: 2 n-6)$ & 19.92 & 0.259 & $-0.6^{\star}$ & 0.221 & 18.96 & 0.266 & -0.61 & 0.311 & $0.011^{*}$ & $0.040^{*}$ & 0.969 & 0.978 \\
\hline$n-6$ PUFA & GLA $(18: 3 n-6)$ & 0.2 & 0.01 & 0.01 & 0.012 & 0.23 & 0.014 & 0 & 0.012 & $0 \cdot 101$ & $0 \cdot 182$ & 0.669 & 0.807 \\
\hline$n-6$ PUFA & Eicosadienoic $(20: 2 n-6)$ & 0.22 & 0.004 & 0 & 0.003 & 0.21 & 0.004 & 0 & 0.004 & $0.006^{*}$ & $0.040^{*}$ & 0.431 & 0.807 \\
\hline$n-6$ PUFA & DGLA $(20: 3 n-6)$ & 1.58 & 0.036 & $-0.07^{\star}$ & 0.029 & 1.46 & 0.037 & -0.04 & 0.033 & 0.024 & 0.060 & 0.467 & 0.807 \\
\hline$n-6$ PUFA & ARA $(20: 4 n-6)$ & 8.66 & 0.152 & 0.08 & 0.156 & 8.32 & 0.146 & -0.15 & 0.184 & 0.109 & 0.182 & 0.351 & 0.807 \\
\hline Desaturase index D5D & ARA:DGLA & $5 \cdot 7$ & 0.17 & $0.35^{\star}$ & 0.12 & 5.93 & 0.07 & 0.12 & 0.17 & 0.338 & & 0.102 & \\
\hline \multirow[t]{7}{*}{ Desaturase index D6D } & DGLA:linoleic & 0.08 & 0.002 & 0 & 0.002 & 0.08 & 0.001 & 0.002 & 0.002 & 0.442 & & 0.393 & \\
\hline & SFA & $37 \cdot 2$ & 0.22 & $0.7^{*}$ & 0.294 & 37.63 & 0.255 & 0.38 & 0.594 & 0.203 & & 0.639 & \\
\hline & MUFA & 22.07 & 0.299 & -0.21 & 0.256 & 23.07 & 0.282 & $-0.83^{\star}$ & 0.372 & $0.016^{\star}$ & & 0.166 & \\
\hline & PUFA & 36 & 0.338 & -0.21 & 0.361 & 34.66 & 0.365 & -0.63 & 0.565 & $0.008^{\star}$ & & 0.533 & \\
\hline & PUFA $(n-3)$ & 5.43 & 0.156 & 0.37 & 0.2 & 5.46 & 0.155 & 0.23 & 0.2 & 0.872 & & 0.419 & \\
\hline & PUFA $(n-6)$ & 30.58 & 0.315 & -0.57 & 0.317 & 29.18 & 0.313 & -0.43 & 0.317 & $0.002^{*}$ & & 0.747 & \\
\hline & $n-3: n-6$ & 0.18 & 0.006 & -0.017 & 0.004 & 0.19 & 0.006 & 0.009 & 0.004 & 0.181 & & 0.221 & \\
\hline
\end{tabular}

ALA, $\alpha$-linolenic; GLA, $\gamma$-linolenic acid; DGLA, dihomo- - -linolenic; ARA, arachidonic acid; FDR, false discovery rate

* $P$ values are significant at the FDR $5 \%$ level.

$\dagger P$ values were obtained from generalised linear models including the responder group as a factor. FDR-adjusted $P$ values control for FDR. The $\Delta 5$ desaturase was calculated as the ratio of arachidonic acid (20:4n-6):dihomo- - -linoleic acid $(20: 3 n-6)$. The $\Delta 6$ desaturase was calculated as the ratio of dihomo- $\gamma$-linoleic acid $(20: 3 n-6)$ :linoleic acid $(18: 2 n-6)$. 
Table 4. Mean blood carotenoid levels $(\mu \mathrm{mol} / \mathrm{l})$ for responders and non-responders at baseline $\dagger$ (Carotenoid levels at baseline and mean change $(\Delta)$ between baseline and month 6 are presented as means with their standard errors)

\begin{tabular}{|c|c|c|c|c|c|c|c|c|c|c|}
\hline & \multicolumn{4}{|c|}{ Responders } & \multicolumn{4}{|c|}{ Non-responders } & & \\
\hline & \multicolumn{2}{|c|}{ Baseline } & \multicolumn{2}{|c|}{$\Delta$} & \multicolumn{2}{|c|}{ Baseline } & \multicolumn{2}{|c|}{$\Delta$} & \multicolumn{2}{|c|}{$P$ value for difference } \\
\hline & Mean & SEM & Mean & SEM & Mean & SEM & Mean & SEM & Baseline & $\Delta$ \\
\hline Lutein & 0.23 & 0.013 & $-0.03^{*}$ & 0.012 & 0.25 & 0.014 & $-0.03^{*}$ & 0.01 & 0.328 & 0.74 \\
\hline Zeaxanthin & 0.06 & 0.004 & $-0.02^{\star}$ & 0.004 & 0.05 & 0.003 & $-0.01^{\star}$ & 0.003 & 0.525 & 0.282 \\
\hline$\beta$-Cryptoxanthin & 0.24 & 0.028 & $-0.08^{\star}$ & 0.02 & 0.19 & 0.018 & -0.01 & 0.017 & 0.098 & $0.022^{*}$ \\
\hline$a$-Carotene & 0.14 & 0.014 & -0.01 & 0.018 & 0.11 & 0.011 & 0.01 & 0.008 & 0.146 & 0.448 \\
\hline$\beta$-Carotene & 0.45 & 0.035 & -0.05 & 0.034 & 0.4 & 0.035 & 0.02 & 0.021 & 0.276 & 0.098 \\
\hline Lycopene & 0.55 & 0.027 & $-0.07^{\star}$ & 0.026 & 0.54 & 0.033 & -0.01 & 0.038 & 0.863 & 0.225 \\
\hline Total carotenoids & 1.67 & 0.078 & $-0.21^{*}$ & 0.072 & 1.54 & 0.08 & -0.03 & 0.071 & 0.263 & 0.082 \\
\hline
\end{tabular}

${ }^{*} P$ values are significant at the $5 \%$ level.

$\dagger P$ values were obtained from generalised linear models containing the responder group as a factor.

Table 5. Examining the ability to classify responders and nonresponders*

(Area under the ROC curves with their standard errors)

\begin{tabular}{lccccc}
\hline & & & \multicolumn{3}{c}{ Asymptotic } \\
& Area & SE & $P \dagger$ & $95 \% \mathrm{Cl}$ & $P \ddagger$ \\
\hline $\begin{array}{l}\text { M1: anthropometric data } \\
\text { only }\end{array}$ & 0.61 & 0.045 & 0.014 & $0.53,0.70$ & \\
$\begin{array}{l}\text { M2: M1 plus baseline } \\
\quad \text { cholesterol }\end{array}$ & 0.76 & 0.037 & $<0.001$ & $0.69,0.836$ & 0.0007 \\
$\begin{array}{l}\text { M3: M2 plus dietary } \\
\text { intake data }\end{array}$ & 0.76 & 0.037 & $<0.001$ & $0.69,0.836$ & 0.999 \\
$\begin{array}{l}\text { M4: M3 plus biochemical } \\
\text { data }\end{array}$ & 0.90 & 0.026 & $<0.001$ & $0.85,0.95$ & 0.0003 \\
\end{tabular}

AUC, area under the ROC curve.

* The area measures the accuracy, or discrimination ability, to classify responders and non-responders.

$\dagger$ Null hypothesis: true area $=0.5$

$\ddagger P$ value for comparison of $C$-statistic $v$. previous model.

achieve a sensitivity of $80 \%$ in model 3 , the false-positive rate was only $10 \%$. This compares with $67 \%$ for model 1 and $44 \%$ for model 2 (Fig. 1). Furthermore, it is also worth noting that the intervention group was not selected as a discriminant variable, indicating that it did not contribute to classification as a responder or non-responder.

\section{Discussion}

Identification of sub-phenotypes that respond differently to dietary interventions has the possibility to significantly enhance delivery of personalised nutrition. In the present study, a baseline phenotype characterised by age, alcohol intake, and levels of stearic acid, DPA, EPA, eicosenoic acid and trans-FA was identified, which could discriminate responders and non-responders in $90 \%$ of cases. Discriminant analysis has previously been used in dietary intervention studies to test whether metabolic profiles may be used to identify responders and non-responders. In a choline-depletion study, analysis of the baseline metabolomics profile predicted that participants developed liver dysfunction when deprived of dietary choline ${ }^{(23)}$. Mutch et $a l^{(24)}$ classified responders and

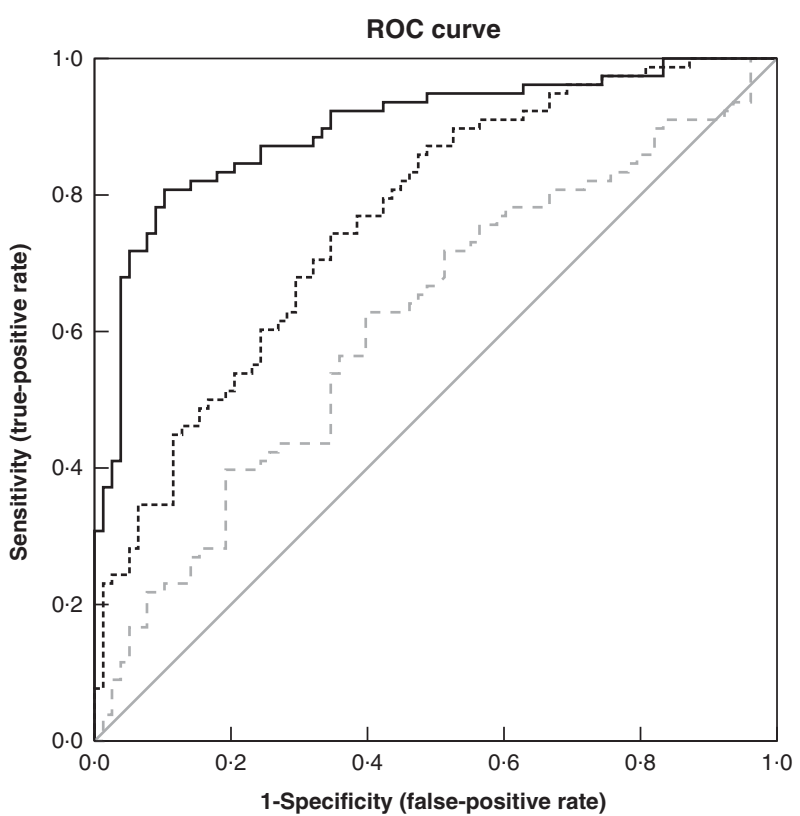

Figure 1. Area under the ROC curves illustrating the performance of models $\mathrm{M} 1, \mathrm{M} 2$ and M4 at discriminating responders from non-responders. The selected variables in $\mathrm{M} 3$ were identical to $\mathrm{M} 2$, and therefore have not been included. The diagonal reference line represents random discrimination, with points above the line indicating discrimination ability.,$--- \mathrm{M} 1$ : anthropometric; -...... M2: M1 + baseline cholesterol; —_, M4: M2 + biochemical; _ reference line.

non-responders to dietary intervention using linear discriminant analysis on a gene expression snapshot. In this study, we used a step-wise logistic regression model to select the individual factors that best classified the probability of being a responder. Incorporation of such information into dietary advice strategies has the potential to significantly enhance the success of interventions.

Wide inter-individual variation has been observed in the response of total, LDL- and HDL-cholesterol to dietary changes ${ }^{(25-27)}$, with little alterations in blood cholesterol for some participants despite significant changes in dietary FA pattern and cholesterol intake ${ }^{(28)}$. This means that, although the population response to a diet can be estimated, the responsiveness a single individual will have as a result of dietary 
Table 6. List of discriminating parameters (Estimates and standard errors)

\begin{tabular}{llrrrl}
\hline & & Estimate & SE & $t$ Value & $P$ \\
\hline & Constant & 30.56 & 6.347 & 23.17 & 0.001 \\
& Baseline cholesterol & 2.95 & 0.583 & 25.55 & 0.001 \\
& Baseline glucose & 1.02 & 0.354 & 8.34 & 0.10 \\
& Age & -0.06 & 0.0232 & 6.67 & 0.016 \\
SFA & Stearic acid $(18: 0)$ & 0.62 & 0.253 & 6.03 & 0.025 \\
MUFA & Eicosenoic acid $(20: 1)$ & 13.53 & 5.16 & 6.88 & 0.007 \\
$n-3$ PUFA & DPA (22:5n-3) & 4.51 & 1.04 & 18.76 & 0.001 \\
$n-6$ PUFA & EPA (20:5n-3) & -2.73 & 0.717 & 14.53 & 0.001 \\
& Trans-fatty acids & -3.03 & 1.054 & 8.27 & 0.010 \\
& Alcohol intake & 0.0033 & 0.0011 & 8.25 & 0.042 \\
& & & & &
\end{tabular}

* Step-wise logistic regression discriminant analysis. Estimates are on the logit scale This is the final model selected using step-wise selection procedure including all demographic, anthropometric and dietary intake data as well as fatty acid and carotenoid intakes as potential predictors. The logistic regression model estimates the probability of being a responder. A positive coefficient for an independent variable implies an increased probability of being a responder with increasing values of the variable.

change is difficult to determine ${ }^{(29)}$. Lefevre $e t$ al. ${ }^{(30)}$ observed that variability in the change of serum was related to anthropometric measurements including BMI, waist circumference and body fat percentage. Furthermore, there is a large body of evidence to support the genetic influence on response of plasma cholesterol to dietary interventions ${ }^{(25,31-33)}$. The present study determined a profile that was responsive to dietary advice in terms of lowering cholesterol levels. Overall, this study in conjunction with the literature provides compelling evidence that individual variation and response to interventions have to be incorporated into dietary advice strategies.

The most marked differences between the responder and non-responder phenotypes were found in their baseline FA profiles. The responders had a lower mean percentage of trans-FA at baseline. Trans-FA have been found to increase LDL-cholesterol and decrease HDL-cholesterol levels ${ }^{(34)}$. Although the responders and non-responders did not differ in their total percentage of SFA, contributions of different SFA differed. The responders had lower palmitic acid $(16: 0)$ and higher stearic acid (18:0) than the non-responders. A review comparing the risk factors for stearic acid with other SFA ${ }^{(35)}$ reported that diets high in stearic acid have favourable effects on LDL-cholesterol compared with palmitic acid. However, it has also been reported that stearic acid itself has no cholesterolenhancing effect in clinically very well controlled exchange of single FA, whereas palmitic, myristic and lauric acids have strong cholesterol-raising effects ${ }^{(36)}$

The responder group had lower total MUFA, particularly palmitoleic acid $(16: 1 n-7)$ and cis-vaccenic acid $(18: 1 n-7)$. A meta-analysis investigating the effects of MUFA on cardiovascular and diabetic risk factors observed no consistent evidence for a relationship between MUFA and total cholesterol $^{(36,37)}$. The PUFA profiles differed between the responders and the non-responders, with a more marked difference in the $n-6$ PUFA. The responders had higher levels of linoleic acid $(18: 2 n-6)$ and eicosadienoic acid $(20: 2 n-6)$ compared with the non-responders at baseline. Linoleic acid, the primary $n-6$ PUFA, has been shown to have a cholesterol-lowering effect ${ }^{(36,38)}$, and a recent meta-analysis reported a lower risk of CHD events and deaths with increasing linoleic acid intake ${ }^{(39)}$. Although the total $n$-3 PUFA did not differ between the two groups, the responders had a higher percentage of DPA $(22: 5 n-3)$. Higher levels of DPA in human blood have been shown to be correlated with lower cholesterol $^{(40)}$. Overall, the data support the growing evidence that FA patterns as opposed to single individual FA are important in determining health. Moreover, it supports the importance of adequate intake of PUFA.

The demographic profiles of the responders and nonresponders did not differ, and at baseline the groups also had similar anthropometric characteristics. Dietary intake at baseline was similar across the two groups, with only alcohol intake differing. As this was a study of the effects of personalised nutrition, the dietary advice given to the participants differed between individuals. However, for all the participants, the percentage of subjects receiving dietary advice for specific target nutrients was generally similar. The strengths of this study included the fact that it was a multi-country group with multiple time points allowing analysis of change in response to the intervention. Furthermore, the participants were well phenotyped. A limitation of the present study is the unique study design involving personalised nutrition advice, which makes replication and prospective analysis in an independent cohort difficult.

An objective of this study was to investigate whether the different types of data were useful in classifying whether an individual will respond to the dietary intervention. Our study has shown that baseline phenotypic data provided more classification power than anthropometric or dietary intake data in classifying responsiveness to personalised dietary advice. While the work identified particular predictive characteristics, it was not our aim to establish causative relationships between the variables. Our study has shown that, in principle, we can predict a priori whether an individual's health status will improve in response to the consumption of a given food/diet. This strengthens the evidence base for the concept that intervention and dietary advice can be personalised with more confidence. Future work should examine the optimal method for incorporation of such data into dietary advice and should pave the way for precision nutrition.

\section{Acknowledgements}

This project was supported by the European Commission under the Food, Agriculture, Fisheries and Biotechnology Theme of the 7th Framework Programme for Research and Technological Development, grant no. 265494.

The authors' contributions were as follows: L. K., L. B., E. R. G. and M. C. W. derived the research question for this manuscript, drafted the manuscript and conducted statistical analysis; J. C. M. was the study director of the proof-of-principle study of Food4Me; H. D., I. T., C. A. D., M. G., J. A. L., Y. M., J. A. M. and W. H. M. S. contributed to the design of the proof-of-principle study and were principal investigators for their respective research centre; L. B., R. F., H. F., E. R. G., M. G., S. K., K. M. L., C. F. M. M., C. C.-M., G. M., S. N.-C., C. B. O.'D., A. S., M. C. W. and $\mathrm{C}$. W. contributed to the study design and execution at the 
research centres. All authors read and approved the final version of the manuscript.

C. A. D. is the founder, stock owner, board member and consultant for Vitas Ltd, Oslo, Norway. The other authors have no potential financial or personal conflicts of interest to declare.

\section{Supplementary material}

To view supplementary material for this article, please visit https://doi.org/10.1017/S0007114516004256

\section{References}

1. de Roos B (2013) Personalised nutrition: ready for practice? Proc Nutr Soc 72, 48-52.

2. van Ommen B, Keijer J, Kleemann R, et al. (2008) The challenges for molecular nutrition research 2: quantification of the nutritional phenotype. Genes Nutr 3, 51-59.

3. Konstantinidou V, Ruiz LAD \& Ordovás JM (2014) Personalized Nutrition and Cardiovascular Disease Prevention: from Framingham to PREDIMED. Adv Nutr 5, 368S-371S.

4. Zeevi D, Korem T, Zmora $\mathrm{N}$, et al. (2015) Personalized nutrition by prediction of glycemic responses. Cell $\mathbf{1 6 3}$, 1079-1094.

5. Lampe JW, Navarro SL, Hullar MA, et al. (2013) Interindividual differences in response to dietary intervention: integrating omics platforms towards personalised dietary recommendations. Proc Nutr Soc 72, 207-218.

6. Schork NJ (2015) Personalized medicine: time for oneperson trials. Nature 520, 609-611.

7. Brennan L (2015) Metabotyping: moving towards personalised nutrition. In Metabolomics as a Tool in Nutrition Research, Woodhead Publishing Series in Food Science, Technology and Nutrition, pp. 137-144 [J van Duynhoven, PS Belton, GA Webb and $\mathrm{H}$ van As, editors]. London: RSC Publishing.

8. Kaput J \& Morine M (2012) Discovery-based nutritional systems biology: developing N-of-1 nutrigenomic research. Int J Vitamin Nutr Res 82, 333-341.

9. Celis-Morales C, Lara J \& Mathers JC (2015) Personalising nutritional guidance for more effective behaviour change. Proc Nutr Soc 74, 130-138.

10. Curtis PJ, Adamson AJ \& Mathers JC (2012) Effects on nutrient intake of a family-based intervention to promote increased consumption of low-fat starchy foods through education, cooking skills and personalised goal setting: the Family Food and Health Project. BrJ Nutr 107, 1833-1844.

11. Ryan NM, O'Donovan CB, Forster H, et al. (2015) New tools for personalised nutrition: the Food4Me project. Nutr Bull $\mathbf{4 0}$, 134-139.

12. O'Sullivan A, Gibney MJ, Connor AO, et al. (2011) Biochemical and metabolomic phenotyping in the identification of a vitamin D responsive metabotype for markers of the metabolic syndrome. Mol Nutr Food Res 55, 679-690.

13. van Bochove K, van Schalkwijk DB, Parnell LD, et al. (2012) Clustering by plasma lipoprotein profile reveals two distinct subgroups with positive lipid response to fenofibrate therapy. PLOS ONE 7, e38072.

14. Elnenaei MO, Chandra R, Mangion T, et al. (2011) Genomic and metabolomic patterns segregate with responses to calcium and vitamin D supplementation. Br J Nutr 105, 71-79.

15. Rudkowska I, Paradis AM, Thifault E, et al. (2013) Differences in metabolomic and transcriptomic profiles between responders and non-responders to an $n-3$ polyunsaturated fatty acids (PUFAs) supplementation. Genes Nutr $\mathbf{8}$, 411-423.

16. Celis-Morales C, Livingstone KM, Marsaux CF, et al. (2015) Design and baseline characteristics of the Food4Me study: a web-based randomised controlled trial of personalised nutrition in seven European countries. Genes Nutr 10, 450.

17. Celis-Morales C, Livingstone KM, Woolhead C, et al. (2015) How reliable is internet-based self-reported identity, sociodemographic and obesity measures in European adults? Genes Nutr 10, 1-10.

18. Baecke JA, Burema J \& Frijters JE (1982) A short questionnaire for the measurement of habitual physical activity in epidemiological studies. Am J Clin Nutr 36, 936-942.

19. Marsaux CFM, Celis-Morales C, Livingstone KM, et al. (2016) Changes in physical activity following a genetic-based internet-delivered personalized intervention: randomized controlled trial (Food4Me). J Med Internet Res 18, e30.

20. Forster H, Fallaize R, Gallagher C, et al. (2014) Online dietary intake estimation: the Food4Me food frequency questionnaire. J Med Internet Res 16, e150.

21. Fallaize R, Forster H, Macready AL, et al. (2014) Online dietary intake estimation: reproducibility and validity of the Food $4 \mathrm{Me}$ food frequency questionnaire against a 4-day weighed food record. J Med Internet Res 16, e190.

22. Hoeller U, Baur M, Roos FF, et al. (2016) Application of dried blood spots to determine vitamin D status in a large nutritional study with unsupervised sampling: the Food4Me project. Br J Nutr 115, 202-211.

23. Sha W, da Costa KA, Fischer LM, et al. (2010) Metabolomic profiling can predict which humans will develop liver dysfunction when deprived of dietary choline. FASEB J $\mathbf{2 4}$, 2962-2975.

24. Mutch DM, Temanni MR, Henegar C, et al. (2007) Adipose gene expression prior to weight loss can differentiate and weakly predict dietary responders. PLOS ONE 2, e1344.

25. Masson LF, McNeill G \& Avenell A (2003) Genetic variation and the lipid response to dietary intervention: a systematic review. Am J Clin Nutr 77, 1098-1111.

26. Beynen AC, Katan MB \& Van Zutphen LF (1987) Hypo- and hyperresponders: individual differences in the response of serum cholesterol concentration to changes in diet. Adv Lipid Res 22, 115-171.

27. Jacobs DR, Anderson JT, Hannan P, et al. (1983) Variability in individual serum cholesterol response to change in diet. Arteriosclerosis 3, 349-356.

28. Cox C, Mann J, Sutherland W, et al. (1995) Individual variation in plasma cholesterol response to dietary saturated fat. BMJ 311, 1260-1264.

29. Denke MA, Adams-Huet B \& Nguyen AT (2000) Individual cholesterol variation in response to a margarine- or butterbased diet: a study in families. JAMA 284, 2740-2747.

30. Lefevre M, Champagne CM, Tulley RT, et al. (2005) Individual variability in cardiovascular disease risk factor responses to low-fat and low-saturated-fat diets in men: body mass index, adiposity, and insulin resistance predict changes in LDL cholesterol. Am J Clin Nutr 82, 957-963; quiz $1145-1146$.

31. Qi Q, Durst R, Schwarzfuchs D, et al. (2015) CETP genotype and changes in lipid levels in response to weight-loss diet intervention in the POUNDS LOST and DIRECT randomized trials. J Lipid Res 56, 713-721.

32. Asztalos B, Lefevre M, Wong L, et al. (2000) Differential response to low-fat diet between low and normal HDLcholesterol subjects. J Lipid Res 41, 321-328. 
33. Wallace AJ, Mann JI, Sutherland WH, et al. (2000) Variants in the cholesterol ester transfer protein and lipoprotein lipase genes are predictors of plasma cholesterol response to dietary change. Atherosclerosis 152, 327-336.

34. Hunter JE (2014) Health and nutrition update on trans fatty acids. Lipid Technol 26, 199-201.

35. Hunter JE, Zhang J \& Kris-Etherton PM (2010) Cardiovascular disease risk of dietary stearic acid compared with trans, other saturated, and unsaturated fatty acids: a systematic review. Am J Clin Nutr 91, 46-63.

36. Müller H, Kirkhus B \& Pedersen JI (2001) Serum cholesterol predictive equations with special emphasis on trans and saturated fatty acids. An analysis from designed controlled studies. Lipids 36, 783-791.

37. Schwingshackl L \& Hoffmann G (2012) Monounsaturated fatty acids and risk of cardiovascular disease: synopsis of the evidence available from systematic reviews and metaanalyses. Nutrients 4, 1989-2007.

38. Harris WS, Mozaffarian D, Rimm E, et al. (2009) Omega-6 fatty acids and risk for cardiovascular disease: a Science Advisory from the American Heart Association Nutrition Subcommittee of the Council on Nutrition, Physical Activity, and Metabolism; Council on Cardiovascular Nursing; and Council on Epidemiology and Prevention. Circulation 119, 902-907.

39. Farvid MS, Ding M, Pan A, et al. (2014) Dietary linoleic acid and risk of coronary heart disease: a systematic review and meta-analysis of prospective cohort studies. Circulation 130, $1568-1578$

40. Byelashov OA, Sinclair AJ \& Kaur G (2015) Dietary sources, current intakes, and nutritional role of omega-3 docosapentaenoic acid. Lipid Technol 27, 79-82. 